\title{
Levels, Trends and Inter-Regional Variations in Transfusion Transmissible Infection Positivity among Blood Donors in India: Evidence from India's National HIV Program
}

\author{
Saravanamaurthy P. Sakthivel1, Pinnamaneni Yujwal Raj², Shanoo Mishra ${ }^{3}$, \\ Arun Kumar Sharma ${ }^{4}$ \\ ${ }^{1}$ National AIDS Control Organization, Ministry of Health and Family Welfare, \\ New Delhi, India \\ ${ }^{2}$ Strategic Information Management Unit, National AIDS Control Organization, \\ Ministry of Health and Family Welfare, New Delhi, India \\ ${ }^{3}$ Blood Safety Division, National AIDS Control Organization, Ministry of Health and Family Welfare, \\ New Delhi, India \\ ${ }^{4}$ Department of Community Medicine, University College of Medical Sciences, New Delhi, India \\ Email: pssaravanamurthy@gmail.com
}

Received 20 August 2015; accepted 22 September 2015; published 25 September 2015

Copyright (C) 2015 by authors and Scientific Research Publishing Inc.

This work is licensed under the Creative Commons Attribution International License (CC BY).

http://creativecommons.org/licenses/by/4.0/

(c) (i) Open Access

\section{Abstract}

In India, transfusion transmissible infections (TTIs) are monitored for trends at the blood bank level, but limited efforts have been made to analyze data from a large number of blood banks and present it at the national, regional and state levels. The Computerized Management Information System (CMIS), National AIDS Control Organization's (NACO) online data capturing system, systematically collects information on TTIs in a monthly aggregated format from blood banks across the country. This information is being captured in NACO's annual reports, but a systematic scientific analysis and publication of the same have not been attempted so far. Hence, the objective of this study is to analyze and report TTIs among blood donors in India at three different levels-national, regional and state-from 497 consistent blood banks reporting through CMIS during 20082012. National level analysis shows an increasing trend in voluntary blood donation from 9.28 lakh to 19.02 lakh, and a decreasing trend in TTIs, with female donors constituting only $6 \%$ of the entire pool of donors. Of the five TTIs studied, in 2012 Hepatitis-B-surface Antigen (HBsAg) positivity was highest $(0.9 \%)$, followed by Hepatitis C Virus (HCV) $(0.45 \%)$, syphilis $(0.23 \%)$, HIV $(0.17 \%)$ and malaria $(0.03 \%)$. Regional analysis indicated that voluntary donation was high in the Eastern and Western regions, with $11 \%$ of female donors in Eastern India in 2012. All the TTIs showed a declining trend, except HCV, which showed a rising trend in the Eastern and Southern 
regions. The level of positivity for all the five TTIs was higher than the national average in the Eastern region. Among the TTIs, HIV positivity among voluntary donors was below the national average, while in Mizoram both HBsAg and HCV of more than 1\% sero-positivity and VDRL of 1.7\% in Arunachal Pradesh had been reported. Malaria has the lowest national positivity, with Nagaland reporting a high positivity of $0.4 \%$. Although the national and regional trends in TTI positivity are declining, regional level variations in TTIs, especially in hepatitis, suggest the importance of advocating for pre-donation counseling and educating donors on self-exclusion.

\section{Keywords}

\section{HIV, Hepatitis, Transfusion Transmissible Infections, CMIS, Female Donors, India}

\section{Background}

Globally, World Health Organization (WHO) has recommended mandatory screening of all donated blood units for transfusion transmissible infections (TTIs), including HIV, Hepatitis B, Hepatitis C and syphilis, prior to usage. Out of 160 countries worldwide, 39 countries do not even routinely screen for TTIs; the irregular supply of test kits is a key barrier to regular TTI screening [1].

In India, as a part of ensuring safe blood transfusion, all blood units are being screened for five major infections, including HIV, Hepatitis-B surface Antigen (HBsAg), Hepatitis C virus (HCV), syphilis and malaria. Following the first report of blood transfusion associated HIV in 1987, screening of donated blood for HIV and HBsAg was made mandatory in 1989 across the country. The diagnostic methodology in India ranges from rapid tests to Nucleic Acid Amplification Tests (NAT), depending upon the resources available [2]. These differences in testing methodology have resulted in the diagnosis of infection status with varying window periods, which do not allow a common interpretation of TTI results across different blood banks in the country. Most previous studies on TTIs in India have not been able to provide national level or even regional level analyses, but instead cover a limited number of blood banks [3]-[6]. A 10-year (2000-2009) study in a tertiary care center blood bank in Pune, India reported a significant decrease in transfusion transmissible viral infections, including HIV, HBsAg and HCV, among 51\% replacement and 49\% voluntary blood donors [3]. Another 5-year (2008-2012) study in a blood bank in Lucknow, India, also reported declining TTIs among blood donors, with replacement donors having higher TTIs than voluntary donors [4].

Considering the importance of tracking the trend of TTIs in donated blood units in the country, the National AIDS Control Organization (NACO), during National AIDS Control Program Phase-II (NACP-II), introduced the Computerized Management Information System (CMIS). This online reporting system maintains the data reported by program implementation units in an aggregated format. The data can be segregated at the implementation unit level, and at the district, state and national levels. Blood banks run by the government, private agencies and NGOs across the country are enrolled in this online system.

In 2003, India's national blood policy proposed to inform, educate, motivate, recruit and retain blood donors to ensure the availability of infection-free blood and blood products [7]. To this end, several campaigns have been organized in the country to increase voluntary blood donation, and thereby reducing replacement donors. This sustained effort has increased voluntary blood donation in the country from 20\% in 1999 to 50\% in 2005 [8].

Considering the changing policies in the country, and additionally, the changing trends in TTIs, it is essential to periodically examine the patterns of TTIs among donors. Hence, the objectives of this study are three-fold: 1 ) to examine patterns of blood donation, types of donors and TTI positivity trends and levels at the national level in India during 2008-2012; 2) to analyze and compare the patterns of blood donation, type of donors and TTI positivity trends and levels across different regions in India during 2008-2012; and finally, 3) to understand the TTI positivity level in various states of India during 2008-2012.

\section{Data and Methods}

\subsection{Data}

CMIS blood bank data from NACO for the period 2008-2012 were used in this study due to change of online 
reporting format from 2013 and very less number of blood banks and from few states had reported before 2008. The data were cleaned for non-reporting units, duplicate units and outliers. This is aggregated monthly data, and the smallest unit of data available is at the blood bank level. Data from 29 states and Union Territories, covering 246 districts and 497 blood banks, as per the eligibility criteria, have been considered in this analysis.

\subsection{Eligibility Criteria}

Blood banks that have been consistently reporting $\geq 9$ months data for all the five years (2008-2012) have been included in the analysis.

\subsection{Variables}

The variables included in the analysis are gender of the donor (male or female), type of donor (voluntary or replacement), and positivity status with regard to HIV, HBV, HCV, syphilis and malaria. The findings are presented by levels and trends; trends cover 2008-2012, whereas levels of positivity are presented based on 2012 data.

Findings are presented at three levels: national, regional and state. National covers all the blood banks that have been considered in this study. Regions are sub-divisions of the country, for this study, the five divisions of the country-East, North, North-East, South and West as commonly adopted by NACO [9] are considered. The following states/Union Territories comprise these regions - the Eastern region includes Odisha, West Bengal, Bihar and Jharkhand; the Northern region Chandigarh, Delhi, Haryana, Himachal Pradesh, Punjab, Rajasthan, Uttar Pradesh and Uttarakhand; the North-Eastern region Arunachal Pradesh, Assam, Meghalaya, Mizoram, Nagaland, Sikkim and Tripura; the Southern region Karnataka, Kerala, Tamil Nadu and Puducherry, and the Western region Dadar-Haveli, Daman \& Diu, Goa, Gujarat, Maharashtra and Madhya Pradesh.

For six states_-Jammu and Kashmir (North), Tripura and Manipur (North-East), Chhattisgarh (East) and Andhra Pradesh and Telangana (South) we did not have complete data, as per the eligibility criteria of the study, hence these states are not considered for in the analysis.

\subsection{Statistical Analysis}

Descriptive analyses have been presented to describe TTI positivity by type of blood donor over the five year period. TTI positivity levels, trends at the national level and by type of blood donor have also been presented. Finally, for 2008 and 2012 data, the levels of TTI positivity are presented in proportions by segregating the states into five regions.

\subsection{Ethical Considerations}

The Institutional Review Board of NACO has approved this study, as the data do not have any personal identifiers and no primary data collection was involved.

\section{Results}

\subsection{National Blood Collection: Levels, Trends and Variations}

Annual blood collection in India increased from 19.78 lakh in 2008 to 25.78 lakh in 2012, with a major increase in voluntary donation from 47\% (9.28 lakh) in 2008 to 74\% (19.02 lakh) in 2012 and a steep decrease in replacement donation (Figure 1). Overall, gender-wise donated units remained consistent over the study period, with $94 \%$ male and $6 \%$ female donors at the national level.

At the national level (Figure 2), there has been a decrease in all TTIs among voluntary and replacement donors over the study period. HIV positivity was higher among voluntary donors than replacement donors in 2008, reached the same level in 2009, and remained less in voluntary donors than replacement donors from 2010. HIV and syphilis positivity were $0.17 \%$ and $0.23 \%$ respectively among voluntary donors. The level of HIV, $\mathrm{HBsAg}$ and HCV were similar among voluntary and replacement donors in 2012. In the case of syphilis and malaria, the positivity level was lower among voluntary donors than replacement donors. HCV $(0.45 \%)$ and malaria $(0.03 \%)$ were at the same level throughout the study period. Of the five TTIs, HBsAg was the highest at $0.9 \%$ in 2012 , while malaria was the lowest at $0.03 \%$. HBsAg $(0.9 \%)$ and HCV $(0.45 \%)$ were found to be higher than HIV 


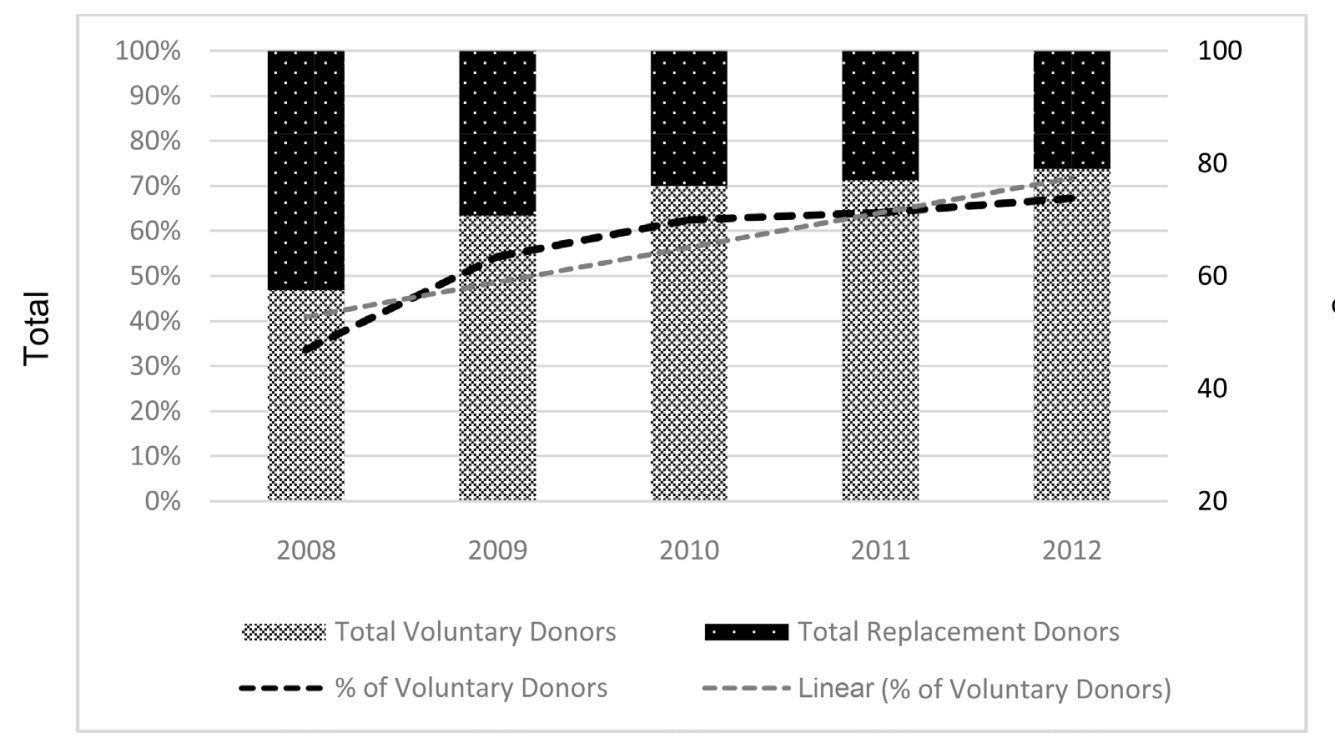

Figure 1. Trends by type of blood donor, India, 2008-2012.

positivity $(0.17 \%)$.

\subsection{Regional Blood Collection: Trends and Levels among Voluntary Donors}

Voluntary blood donation increased across all five regions during the study period. Of the five regions, blood donation in the Eastern region was consistently high. The Eastern and Western regions contributed to the highest proportion of voluntary donations in the country (82\% and 79\%, respectively in 2012). In 2008 voluntary blood donation in the North-East was higher as compared to the South and North, while in 2012, voluntary blood donation in the Northern and Southern regions increased as compared to the North-Eastern region (Figure 3). The Eastern region had the highest percentage of female donors (8\% in 2008 and $10 \%$ in 2012), followed by the North-East (6\% in 2012). The North and West followed a similar level and trend in female donors, while Southern India was the lowest with regard to female donors (reporting $2 \%$ consistently over the study period).

\subsection{Regional TTIs: Trends and Levels among Voluntary Donors}

At the regional level (Figure 4), all TTIs have been decreasing among voluntary blood donors over the study period, apart from HCV which showed a marginal increase.

HIV: HIV showed a decreasing trend in the Eastern region, from $0.6 \%$ to $0.2 \%$, while in other regions it remained at the same level. Of the five regions, the South and North-East have been showing similar levels of HIV positivity throughout the study period. The level of HIV positivity in all the regions was less than the national average (0.17\%), except in the Eastern region, where it was $0.26 \%$.

HBsAg: The trend for HBsAg positivity was similar to that of HIV in all the regions. HBsAg positivity remained consistently around $1 \%$ in the Northern, Eastern and Western regions of the country. The North-Eastern region reported the lowest level of HBsAg positivity. Three regions - the North, East and West reported higher sero-positivity than the national average $(0.97 \%)$, while the South and North-East reported positivity levels lower than the national average.

$H C V$ : HCV positivity showed a flat trend across all regions, except in the North-East where it showed a declining trend. The level of HCV positivity was highest in the East and lowest in the West. The North-East, South and West reported a lower level of HCV positivity than the national HCV positivity level (0.4\%) among voluntary donors. The Northern region had a positivity rate similar to the national level, while the Eastern region showed higher positivity than the national average.

Syphilis: With regard to syphilis, trends and levels were similar in the North-East, North and West, comparatively higher in the East and lower in the South. Syphilis positivity was higher in the East, West and Northern regions as compared to the national average, $0.23 \%$. 

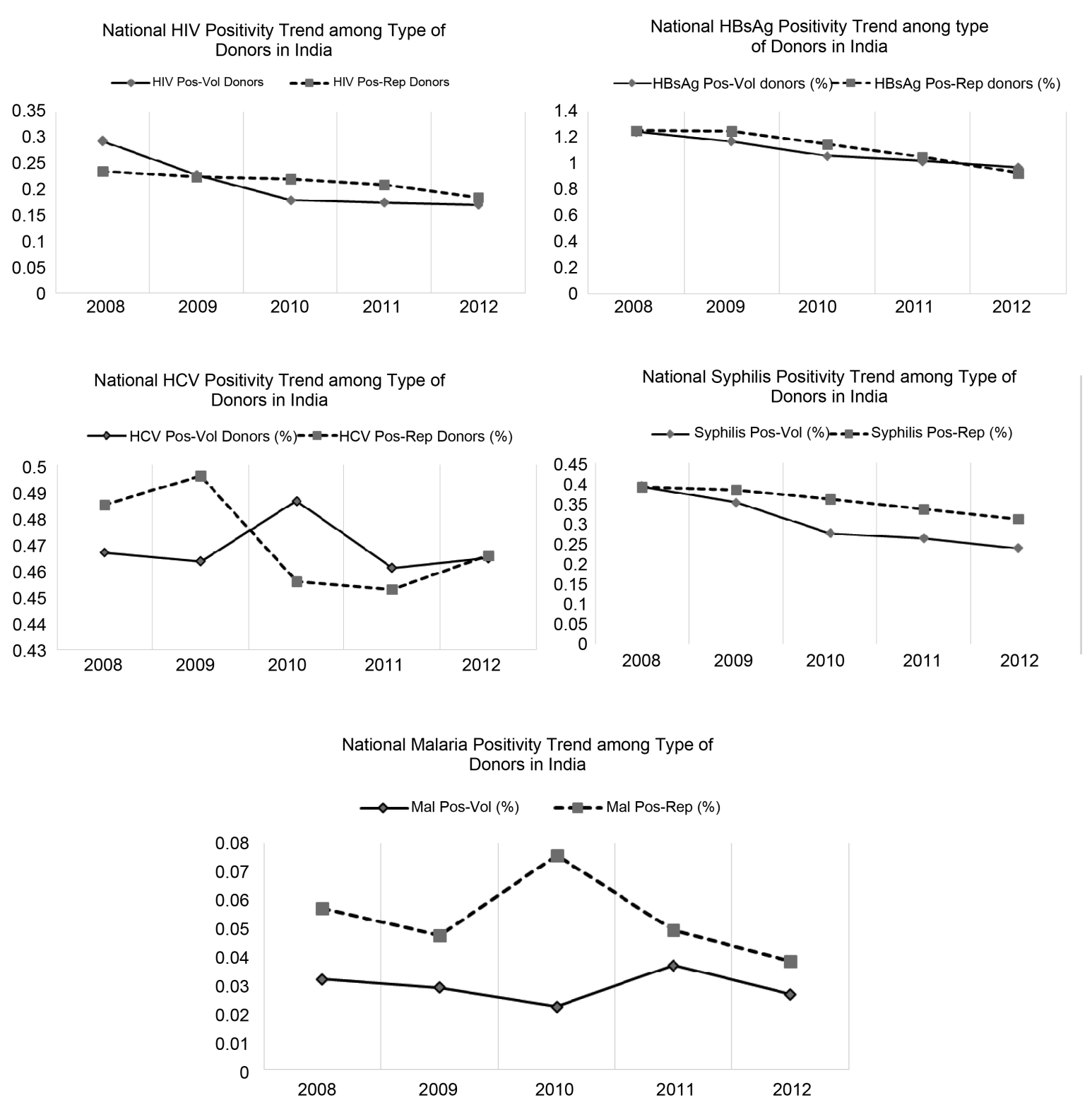

Figure 2. Trends in TTIs among donated blood units by type of donor, India, 2008-2012.

Malaria: The trend in malaria positivity was similar across all regions, except in the West which showed a marginal increase from 2010. The level of malaria positivity was highest in the East and North-East, and lowest in the South. The North-East, East and West had higher malaria positivity than the national average among voluntary donors.

\subsection{Levels of Blood Collection: State}

Of the total blood collected, voluntary blood donation was the highest in Dadra, Nagar and Haveli (100\%) and lowest in Odisha (31\%). Of the 29 states considered in this analysis, 13 states collected less than the national average, and 16 states collected more than the national voluntary blood donation average (73.80\%).

\subsection{TTI Positivity among Voluntary Donors at the State Level}

HIV: Zero HIV positivity was observed in three states—Arunachal Pradesh, Sikkim and Tamil Nadu—in 2012. 


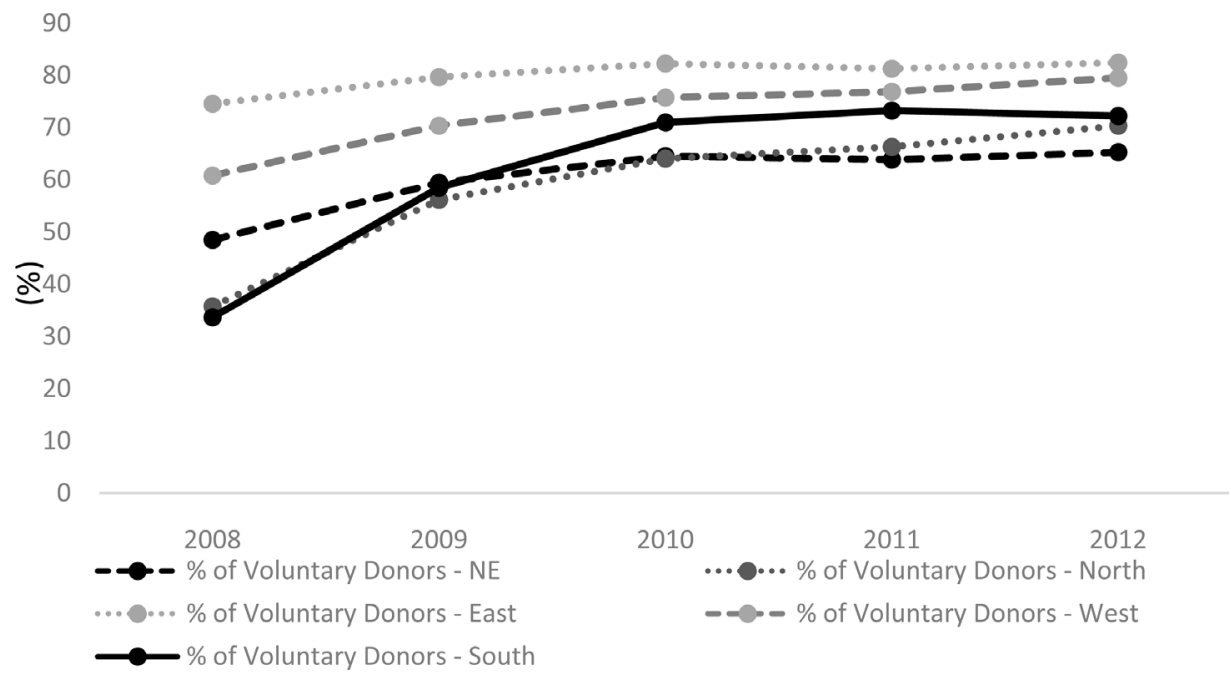

Figure 3. Trends and levels of voluntary donation by region, India, 2008-2012.

West Bengal had 0.31\% positivity, while the remaining states reported lower levels than West Bengal. HIV sero-positivity in all the 29 states was less than the national average for HIV sero-positivity (0.48\%).

Hepatitis-B Surface Antigen: The national average for HBsAg positivity was $0.97 \%$ in 2012 . The states reporting HBsAg positivity higher than the national average were Bihar (1.9\%), Puducherry (1.8\%), Dadara, and Nagar Haveli (1.7\%), Arunachal Pradesh (1.5\%), Mizoram (1.5\%) and Rajasthan (1.3\%).

HCV: Zero HCV positivity was reported in Arunachal Pradesh, Odisha and Tamil Nadu. Six states-Delhi (0.60\%), Haryana (0.73\%), Mizoram (1\%), Punjab (1\%), Uttarakhand $(0.60 \%)$ and West Bengal (0.92\%)—reported higher HCV positivity than the national average (0.4\%).

Syphilis: The national syphilis positivity was $0.23 \%$ among voluntary donors. The states with syphilis positivity higher than the national average were Arunachal Pradesh (1.7\%), Delhi (0.32\%), Madhya Pradesh (0.29\%), Rajasthan (0.31\%) and West Bengal (0.41\%).

Malaria: Among voluntary donors the national positivity for malaria was $0.02 \%$. Nine states reported malaria positivity higher than the national average-Arunachal Pradesh (0.2\%), Nagaland (0.4\%), Sikkim (0.05\%), Bihar (0.2\%), Jharkhand (0.2\%), Uttar Pradesh (0.03\%), Daman and Diu (0.07\%), Madhya Pradesh (0.05\%) and Maharashtra (0.04\%). All the four Southern states—Karnataka, Kerala, Puducherry and Tamil Nadu—had malaria positivity less than the national average.

\section{Discussion}

This retrospective analysis of blood bank data on TTI from India is the first of its kind to cover 497 blood banks across 29 states [3]-[5] [10]. Hence this study provides a better representation across regions in India than earlier studies. The study shows an overall decline in TTIs at the national level among voluntary and replacement donors, while at the regional level all TTIs have been declining among voluntary donors, except HCV. The level of hepatitis infections (both HBsAg and HCV) was found to be higher than HIV at the national level among voluntary and replacement donors. The high positivity levels of the two hepatitis infections in the Eastern and Western regions of the country shows the need for additional attention by the blood safety program in these two regions, rather than the entire country. Studies [11]-[13] have linked hepatitis infections with a history of tattooing and blood transfusion. Our study does not have the scope to explore the profile of donors with Hepatitis infections. Hence, it is suggested that qualitative studies are conducted to explore the reasons for hepatitis infection among blood donors, specifically in the Eastern and Western part of India. In addition, the blood safety program could educate donors on the importance of providing uninfected blood through pre-donation counseling, and self-exclusion of donors based on their infection status, vulnerability to infectious diseases and their history of high-risk behaviors.

Voluntary donation in India has increased considerably, and HIV positivity among blood donors has been reduced to less than $0.5 \%$ at the national, regional and state level, as per the goal of National AIDS Control Program 
HIV Positivity Trend

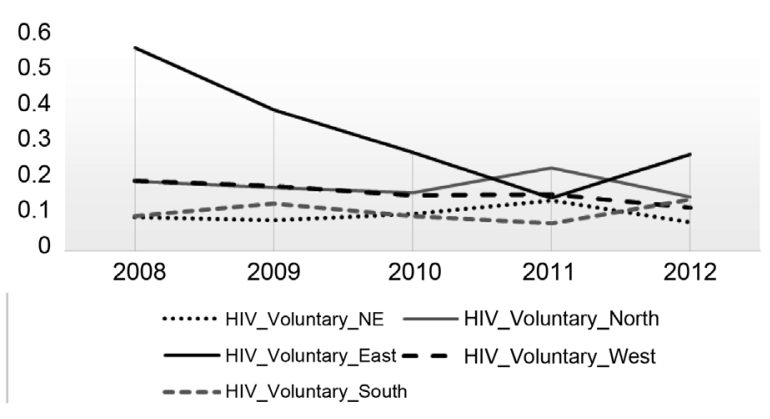

HCV Positivity Trend

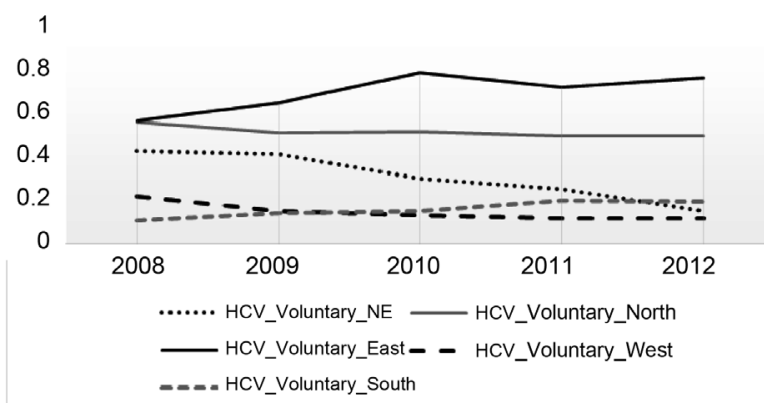

HBsAg Positivity Trend
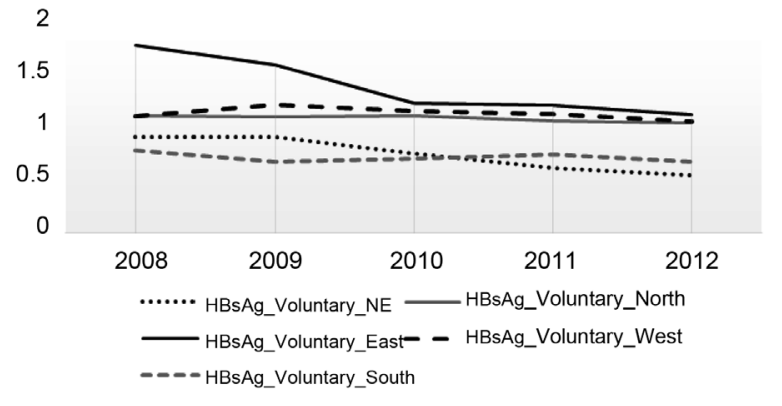

Syphilis Positivity Trend

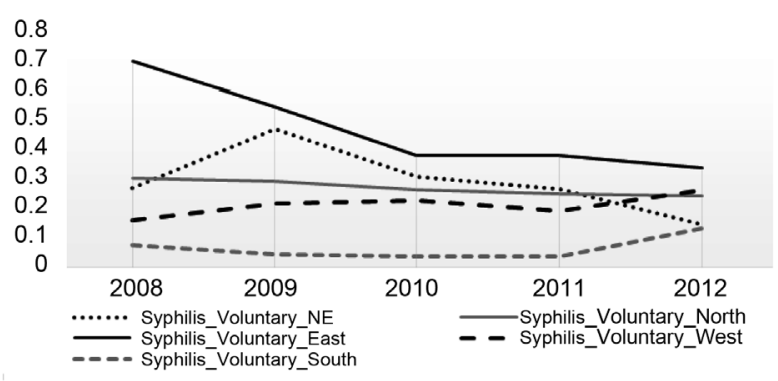

Malaria Positivity Trend

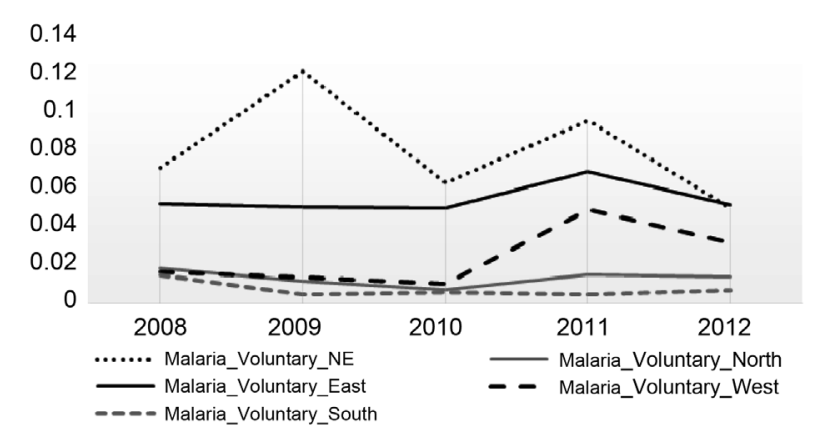

Figure 4. Trends in TTIs in voluntarily donated blood units by region, India, 2008-2012.

III (2007-2012) [8]. This success can be partly attributed to the policies developed by the national program, rigorous implementation of quality standards at the state level, blood banks' adherence to protocols, and increased knowledge/awareness of blood donors [14]-[16]; however, the impact of these factors yet to be studied in India.

This retrospective analysis has established that nationally, female donors are consistently at a very low level; there are variations across regions in female donors, with the Southern region reporting $2 \%$ and the Eastern region reporting $10 \%$. Earlier studies have shown low participation of females in blood donation, and among femaledonors who participate, deferral rates are high [17]-[19]. Low female participation and high rates of deferral among females in India and other countries may be due to several reasons such as women's low weight, anaemia, hypertension and misconceptions about blood donation including loss of energy [20] [21]. Globally, only 20 out of 111 countries have more than $10 \%$ female donors [22]. The present study shows that India has less than $10 \%$ female donors; hence the blood safety program, in collaboration with various other development programs, should address the barriers to females becoming blood donors.

Given the significance of the findings at the national level, similar analyses of programmatic data should be advocated. In addition, the regional level analysis shows a significant difference in TTIs, particularly hepatitis, 
and an in-depth exploration on the reasons for such positivity needs to be explored at the regional level. These findings would help program designers better understand the context.

Limitations: The findings of this study should be interpreted in light of certain limitations. Since six states are not a part of this study, due to the selection criteria, the present findings should not be considered generalized. In addition, the diagnostic tests for TTIs vary between facilities, which results in varying sensitivities and infection status. This study does not explore the facilities based upon the type of diagnostic kits, which is a limitation in understanding the infection status and commenting on the same.

\section{Acknowledgements}

This paper (NACO/SIMU/NDAP/2015/04) was written as part of the National Data Analysis Plan (NDAP), an initiative of the Strategic Information Management Unit of National AIDS Control Organization (NACO), Ministry of Health and Family Welfare, Government of India. We would like to thank the various divisions of NACO and State AIDS Control Organization (SACS) that have collected and maintained the program data. This paper was conceptualized, developed and finalized by the author, under the guidance of mentors (senior public health experts) and the NDAP Unit, NACO. The Knowledge Network project of the Population Council, which is a grantee of the Bill \& Melinda Gates Foundation though Avahan, the India AIDS Initiative, has supported the scientific writing, reviewing, editing and finalization of this paper. This project was also supported by NACO's other development partners, which include Center for Diseases Control (CDC), World Health Organization-India, FHI-360, and John Snow India. The views expressed in this paper are those of the authors, and do not necessarily reflect the views of NACO.

\section{References}

[1] WHO (2011) Blood Safety: Key Global Facts and Figures. World Health Organization, Delhi.

[2] Marwaha, N. and Sachdev, S. (2014) Current Testing Strategies for Hepatitis C Virus Infection in Blood Donors and the Way Forward. World Journal of Gateroenterology, 20, 2948-2954. http://dx.doi.org/10.3748/wjg.v20.i11.2948

[3] Philip, J., Sarkar, R.S., Kumar, S. and Pathak, A. (2012) Changing Trends of Transfusion Transmitted Viral Infections among Blood Donors in the Last Decade-A 10-Year Study in a Large Tertiary Care Blood Bank (2000-2009). Medical Journal of Armed Forces India, 68, 28-32. http://dx.doi.org/10.1016/S0377-1237(11)60125-1

[4] Chandra, T., Rizvi, S.N.F. and Agarwal, D. (2014) Decreasing Prevalence of Transfusion Transmitted Infection in Indian Scenario. The Scientific World Journal, 2014, 4 p.

[5] Sastry, J.M., Agawane, S.U. and Harke, V.A. (2014) Retrospective Study of the Five-Year Prevalence and Trends of Transfusion Transmitted Infections (TTIs) among Blood Donors at a Charitable Hospital Blood Bank In Pune, India. International Journal of Healthcare and Biomedical Research, 2, 193-200.

[6] Giri, P.A., Deshpande, J.D., Phalke, D.B. and Karle, L.B. (2012) Seroprevalence of Transfusion Transmissible Infections among Voluntary Blood Donors at a Tertiary Care Teaching Hospital in Rural Area of India. Journal of Family Medicine and Primary Care, 1, 48-51. http://dx.doi.org/10.4103/2249-4863.94452

[7] National AIDS Control Organization, M.O.H., Governement of India (2003) National Blood Policy. NACO, Delhi.

[8] National AIDS Control Organization, M.O.H., Government of India (2007) Status Note on Blood Safety. NACO, Delhi.

[9] NACO (2015) HIV Sentinel Surveillance 2012-13. A Technical Brief. 2015, National AIDS Control Organisation, Ministry of Health and Family Welfare, Government of India, Delhi.

[10] Kaur, H., Mannan, R. and Manjari, M. (2014) Seroprevalence of the Blood Borne Infection in Blood Donors: Our 11 Year (2001-2011) Experience in a Tertiary Care Teaching Hospital at Amritsar (Punjab). International Journal of Advanced Research, 2, 967-971.

[11] Kabinda, J.M., Akilamalli, T.S., Miyanga, A.S., Donnen, P. and Michelle, D.W. (2015) Hepatitis-B, Hepatitis-C and HIV in Pregnant Women in the Community in the Democratic Republic of Congo. World Journal of AIDS, 5, 124-130. http://dx.doi.org/10.4236/wja.2015.52015

[12] Awadalla, H.I., Ragab, M.H., Nassar, N.A. and Osman, M.A. (2011) Risk Factors of Hepatitis-C Infection among Egyptian Blood Donors. Central European Journal of Public Health, 19, 217-221.

[13] Brandão, A.B.M. and Fuchs, S.C. (2002) Risk Factors for Hepatitis-C Virus Infection among Blood Donors in Southern Brazil: A Case-Control Study. BMC Gastroenterology, 2, 18. http://dx.doi.org/10.1186/1471-230X-2-18

[14] Karim, M.R., Alam, M., Farazi, M.M.R. and Labone, R.J. (2012) Factors Influencing Blood Donation Behavior of 
University Level Students in Bangladesh. Journal of Information Technology, 1, 35-40.

[15] Nigatu, A. and Demissie, D.B. (2014) Knowledge, Attitude and Practice on Voluntary Blood Donation and Associated Factors among Ambo University Regular Students, Ambo Town, Ethiopia. Journal of Community Medicine and Health Education, 4, 315.

[16] Pule, P.I., Rachaba, B., Magafu, M.G.M.D. and Habte, D. (2014) Factors Associated with Intention to Donate Blood: Sociodemographic and Past Experience Variables. Journal of Blood Transfusion, 2014, Article ID: 571678. http://dx.doi.org/10.1155/2014/571678

[17] Sundar, P., Sangeetha, S.K., Seema, D.M., Marimuthu, P. and Shivanna, N. (2010) Pre-Donation Deferral of Blood Donors in South Indian Set-Up: An Analysis. Asian Journal of Transfusion Science, 4, 112-115. http://dx.doi.org/10.4103/0973-6247.67037

[18] Bahadur, S., Jain, S., Goel, R.K., Pahuja, S. and Jain, M. (2009) Analysis of Blood Donor Deferral Characteristics in Delhi, India. South East Asian Journal of Tropical Medicine and Public Health, 40, 1087-1091.

[19] Sareen, R., Gupta, G.N. and Dutt, A. (2012) Donor Awareness: Key to Successful Voluntary Blood Donation. F1000 Research, 1, 29. http://dx.doi.org/10.12688/f1000research.1-29.v1

[20] Bani, M. and Giussani, B. (2010) Gender Differences in Giving Blood: A Review of Literature. Blood Transfusion, 8, 278-287.

[21] Agnihotri, N. (2010) Whole Blood Donor Deferral Analysis at a Center in Western India. Asian Journal of Transfusion Science, 4, 116-122. http://dx.doi.org/10.4103/0973-6247.67035

[22] WHO (2014) About Blood Safety and Availability: WHO. WHO Website. 